\title{
Prediction of bleeding events in patients with venous thromboembolism on stable anticoagulation treatment
}

\author{
Frederikus A. Klok ${ }^{1}$, Volker Hösel ${ }^{2}$, Andreas Clemens ${ }^{1}$, Wilfrid D. Yollo ${ }^{3}$, \\ Clemens Tilke ${ }^{4}$, Sam Schulman ${ }^{5}$, Mareike Lankeit ${ }^{1}$ and \\ Stavros V. Konstantinides ${ }^{1,6}$
}

Affiliations: ${ }^{1}$ Centre for Thrombosis and Haemostasis (CTH), University Medical Centre of the Johannes Gutenberg University, Mainz, Germany. ${ }^{2}$ StatSciConsult, Munich, Germany. ${ }^{3}$ Statistical Programming Pool (S2P), Yaounde, Cameroon. ${ }^{4}$ Dr Tilke Consulting, Schöneck, Germany. ${ }^{5}$ Dept of Medicine, McMaster University and Thrombosis and Atherosclerosis Research Institute, Hamilton, ON, Canada. ${ }^{6}$ Dept of Cardiology, Democritus University of Thrace, Alexandroupolis, Greece.

Correspondence: Stavros V. Konstantinides, Centre for Thrombosis and Haemostasis, University Medical Centre Mainz, Langenbeckstrasse 1, Building 403, 55131 Mainz, Germany.

E-mail: stavros.konstantinidesQunimedizin-mainz.de

ABSTRACT Attempts at identifying patients with an elevated risk of bleeding while on anticoagulation following acute venous thromboembolism (VTE) have largely been unsuccessful thus far. We sought to develop a clinical prediction score for bleeding during stable anticoagulation treatment after acute VTE.

We performed a post hoc analysis of the pooled RE-COVER studies, two double-blind randomised "sister" trials evaluating dabigatran versus standard treatment in 5107 VTE patients.

A score was derived from patients randomised to dabigatran using logistic regression analysis covering the complete follow-up period. The final model, named VTE-BLEED, included six variables and yielded a c-statistic of 0.72 (95\% CI 0.67-0.76). Patients from the derivation cohort in the low-risk group $(<2$ points; $74 \%$ of the derivation population) had a bleeding incidence of $2.8 \%$ compared to $12.6 \%$ in the elevatedrisk group (OR 5.0; 95\% CI 3.5-7.1). The score proved accurate for our primary end-point, i.e. prediction of major bleeding after day 30 ("stable" anticoagulation), both in patients on dabigatran (c-statistic 0.75, 95\% CI $0.61-0.89)$ and those on warfarin $(0.78,95 \%$ CI $0.68-0.86 ; \mathrm{p}=0.77$ for difference).

The new VTE-BLEED score accurately predicted major bleeding events in VTE patients on stable anticoagulation with both dabigatran and warfarin.

$@$ ERSpublications

The new VTE-BLEED score predicted major bleeding events in VTE patients on stable anticoagulation treatment http://ow.ly/TxED300SpwI

Editorial comment in Eur Respir J 2016; 48:1268-1270.

This article has supplementary material available from erj.ersjournals.com

Received: Feb 062016 | Accepted after revision: May 292016 | First published online: July 282016

Support statement: The work of F.A. Klok, M. Lankeit and S.V. Konstantinides was supported by the German Federal Ministry of Education and Research (BMBF 01EO1003). Funding information for this article has been deposited with the Open Funder Registry.

Conflict of interest: Disclosures can be found alongside this article at erj.ersjournals.com

Copyright @ERS 2016 


\section{Introduction}

Current guidelines recommend anticoagulation treatment for 3 months after an episode of venous thromboembolism (VTE) associated with a transient risk factor, and for $\geqslant 3$ months after the first episode of "unprovoked" VTE associated with persistent risk factors or in the absence of a risk factor $[1,2]$. In the latter patient category, the decision to continue or stop anticoagulant treatment after this period should be made by weighing the risk of recurrent VTE once treatment is discontinued against the risk of bleeding during ongoing treatment. In this regard, dependable validated clinical tools for predicting both the thrombotic and the bleeding risk over the long term are crucial for decision making in individual cases. However, importantly, and in contrast to the robust bleeding scores available for patients with atrial fibrillation, no widely accepted prediction rule(s) exist for VTE [1-4].

In the past, attempts to identify independent risk factors for bleeding in patients with VTE resulted in various scores which, for the most part, failed external validation [5-9]. Moreover, widely used bleeding risk scores for patients with atrial fibrillation generally exhibited low accuracy in the VTE setting [8-13]. Of note, a key prerequisite for a successful bleeding prediction rule after acute VTE is its performance in patients on long-term, stable anticoagulation, which may take up to several weeks to achieve [4]. It is this chronic clinical course that will be relevant for the decision whether to stop or continue anticoagulation after the "compulsory" 3-month treatment period. Conversely, early bleeding complications that were included in previous bleeding score studies reflect, at least in part, the acute disease of the patient and the variable initial response to anticoagulant treatment, and may not be relevant for the prediction of bleeding in the long term $[4,14]$.

Based on these considerations, our aim was to develop and test a clinical prediction score for major bleeding during stable anticoagulation treatment for acute VTE. For this purpose, we performed a patient-level post hoc analysis of patients included in the RE-COVER study programme. RE-COVER and RE-COVER II were "sister" studies designed to compare the efficacy and safety of dabigatran etexilate, given $150 \mathrm{mg}$ twice daily, to international normalised ratio (INR)-guided warfarin, both following initial parenteral treatment with unfractionated or low molecular weight heparin for $\geqslant 5$ days $[15,16]$.

\section{Methods \\ Design of the RE-COVER trials}

The study design, inclusion and exclusion criteria, outcome measures and baseline population characteristics of the RE-COVER study programme have been presented in the original reports of these trials $[15,16]$. Briefly, RE-COVER and RE-COVER II had an identical design, being randomised, double-blind, active-control, non-inferiority studies in patients with symptomatic and confirmed proximal deep vein thrombosis (DVT) or acute pulmonary embolism (PE). Patients were followed-up for 6 months for the occurrence of recurrent symptomatic or fatal VTE (primary efficacy outcome) as well as for major or clinically relevant non-major bleeding complications occurring during active treatment (primary safety outcome).

Major bleeding was defined based on the criteria of the International Society on Thrombosis and Haemostasis [17]. Online supplementary appendix 1 demonstrates the definition of clinically relevant non-major bleeding. All bleeding events were adjudicated by independent central committees whose members were unaware of the treatment assignment.

\section{Aim and design of the present study}

The primary objective of our analysis was to derive a robust clinical score for predicting anticoagulantassociated major bleeding in patients on "stable" anticoagulation (defined as the treatment period after the first 30 days) following acute VTE. However, the score was derived from all patients who were included in RE-COVER and RE-COVER II and were randomised to receive dabigatran. The rationale for focusing on the dabigatran (and not the warfarin) population at this first step was based on the following considerations: 1) it takes approximately five half-lives of warfarin to reach steady-state concentrations after initiation of therapy or after dosage adjustments; 2) the steady state of anticoagulation is not achieved until the levels of the vitamin K-dependent clotting factors also reach a new equilibrium; and 3) concomitant early use of parenteral heparin during titration of vitamin $\mathrm{K}$ antagonists generates an additional bleeding risk during the first days of anticoagulation. As a result, and in contrast to the new target-specific oral anticoagulants like dabigatran, it may take several weeks to achieve stable anticoagulation under warfarin $[14,18]$, the first weeks usually not being representative of the entire treatment period with this drug.

For the derivation of a robust clinical score, $\geqslant 10$ events per potential risk factor should be available for the regression analysis. Recognising that the number of bleeding events in the RECOVER study programme was low and with the aim of achieving maximum power for the derivation analysis based on an appropriate large number of bleeding events, we further included all major or non-major clinically relevant bleeding events that occurred during the total study period as end-points of the derivation analysis. 
Following the derivation step, we pursued our primary end-point by evaluating the new score in the patients receiving "chronic" treatment with dabigatran, and subsequently tested the score in patients receiving "chronic" treatment with warfarin (in both cases after the first 30 days following randomisation).

Further objectives of our study were to test the performance of the newly derived score in patients who presented with DVT versus PE separately. Finally, we compared the predictive accuracy of the derived score to that of existing bleeding scores [5-7, 10-12] (online supplementary appendix 3 ).

\section{Statistical analysis}

Out of all recorded baseline variables in the RE-COVER programme, we first filtered and included in the univariate analysis those variables which were statistically (based on the number of events) and/or medically (as judged by three of the authors: FAK, AC and SK) related to bleeding events (online supplementary appendix 2). To find potential combinations of variables which might predict the bleeding risk, we used classification and regression tree (CART) analyses on the RE-COVER data sets. Combinations of variables that showed a large deviance reduction in the splits of CART analyses and defined larger robust subgroups of patients were added to the initial variable list (online supplementary appendix 2). Next, we calculated univariate odds ratios and p-values for all these variables. To do so, continuous variables were categorised at an optimal threshold. For this purpose, we calculated odds ratios for different splits and used the smallest $\mathrm{p}$-value or took a nearby established clinical split (for example, creatinine clearance $\leqslant 60 \mathrm{~mL} \cdot \mathrm{min}^{-1}$ as calculated using the Cockroft-Gault formula, which indicates at least moderate kidney dysfunction according to the Kidney Disease Outcomes Quality Initiative criteria). Notably, patients with an estimated glomerular filtration rate (eGFR) $<30 \mathrm{~mL} \cdot \mathrm{min}^{-1}$ were excluded from the RECOVER trials and thus also from this analysis. Univariate predictors with a significance level of $\mathrm{p}<0.10$ were included in a multivariate logistic regression model. On 1000 bootstrap samples we performed stepwise selection regression based on a significance level of $\mathrm{p}<0.05$. Variables which were significant in $>50 \%$ of the runs were retained in the final model. Furthermore, we verified the quality of the multivariate models using the Akaike information criterion and Bayesian information criterion and checked method robustness by applying different regression techniques: least absolute shrinkage and selection operator, forward and backward regression.

Based on the final model, we constructed a risk score by assigning points to each predictor according to the regression coefficients, by multiplying them with a common factor (if convenient) and rounding to the nearest integer. The diagnostic quality of the proposed risk score was examined using receiver operator characteristic curve analysis. The bleeding score was considered beforehand to discriminate well in the derivation set if its c-statistic was significantly greater than 0.5 (lower limit of $95 \% \mathrm{CI}>0.50$ ). The score point distribution together with the frequency of bleeding indicates an optimal two-level threshold. An odds ratio with $95 \%$ confidence interval was calculated to quantify the risk differences between the low-risk and elevated-risk categories. In the next step, the c-statistic and odds ratio of the score in dabigatran-treated patients after the first 30 days were determined for major bleeding events only. Subsequently, we evaluated the performance of the newly derived score in predefined patient subgroups and clinical settings as mentioned above by determining, weighing and comparing the c-statistic and odds ratios of the newly derived score between the predefined subgroups and clinical scenarios. Data processing and analyses were performed using SAS software for Windows version 9.2 or later (SAS Institute, Inc., Cary, NC, USA). Where convenient, we used packages from R version 3.0.2 (www.r-project.org).

\section{Results}

Patient population and derivation of the prediction score in patients receiving dabigatran 5107 patients from the RE-COVER and RE-COVER II studies were available for analysis. Of these, 2553 were randomised to receive dabigatran and 2554 to warfarin; the baseline characteristics of the study subjects have been described previously $[15,16]$.

In the dabigatran arm of the pooled study population, $37(1.4 \%)$ patients suffered major bleeding and another $101(4.0 \%)$ patients experienced clinically relevant non-major bleeding events at any time point following randomisation (table 1). The derivation of the new score was based on these 138 patients with events, and on the 2415 patients who remained free of clinically relevant haemorrhage.

CART analyses suggested potentially relevant combinations of variables showing a high association with bleeding events. However, most of the tree results defined small groups of patients and were thus not reliable. Further univariate analyses resulted in three combined variables in addition to the initial single-variable list: male patients with systolic blood pressure $\geqslant 140 \mathrm{mmHg}$ at baseline, patients aged $\geqslant 70$ years with a BMI of $\geqslant 30 \mathrm{~kg} \cdot \mathrm{m}^{-2}$ and male patients aged $\geqslant 70$ years (online supplementary appendix 2). 13 of the variables were associated with bleeding in the univariate analysis $(\mathrm{p}<0.1)$ and were subsequently entered in the multivariate analysis (table 2). The final multivariate model included six independently significant clinical variables (or combinations thereof): active cancer (2 points); male patients 
TABLE 1 Overview of bleeding events in the study population

Major bleeding

Non-major clinically relevant bleeding

\begin{tabular}{llr}
\hline $\begin{array}{l}\text { Dabigatran } \\
\text { Complete period }\end{array}$ & \\
$\quad$ “Stable" anticoagulation & $13(1.4)$ & $101(4.0)$ \\
Warfarin & & $67(2.8)$ \\
$\quad$ Complete period & $51(2.0)$ & $167(6.5)$ \\
“Stable" anticoagulation & $25(1.0)$ & $85(3.5)$
\end{tabular}

Data are presented as $\mathrm{n}(\%)$. The number of patients with events in the present analysis exceeds by two the number $(n=136)$ previously reported in the pooled RE-COVER population [15]. The reason is that in the present study, and in contrast to the previous analysis, we considered all events that occurred after randomisation, regardless of whether the patient received study medication; thus, the two additional bleeding events included here are those that occurred before initiation of the study drug.

with uncontrolled arterial hypertension at baseline (1 point); anaemia (1.5 points); history of bleeding (1.5 points); age $\geqslant 60$ years (1.5 points); and renal dysfunction ( 1.5 points) (table 3$)$. The c-statistic for this newly derived VTE-BLEED score was 0.72 (95\% CI 0.67-0.76), with the lower limit of the 95\% confidence interval largely exceeding the predefined 0.50 threshold for acceptable accuracy (fig. 1). Based on the distribution of bleeds in patients with various scores within the study population, the optimal threshold was set at 2 points, a value which best differentiated between patients with low ( $0-1.5$ points) and elevated $(\geqslant 2$ points) bleeding risk on stable anticoagulation. Using this cut-off, a total of 1880 patients ( $74 \%$ of the pooled RE-COVER population) were classified into the low-risk category (bleeding incidence 2.8\%) and $673(26 \%)$ into the elevated-risk category (bleeding incidence 12.6\%). The calculated odds ratio indicated a 5.0 times (95\% CI 3.5-7.1) higher risk of bleeding for patients belonging to the elevated-risk category compared to the low-risk category.

Performance of the VTE-BLEED score in patients receiving warfarin

In the warfarin arm (2554 patients) of the pooled RE-COVER study population, 51 (2.0\%) patients suffered a major bleed and another $167(6.5 \%)$ patients experienced clinically relevant non-major bleed events at any time point following randomisation (table 1). The c-statistic of the new prediction score was 0.59 (95\% CI $0.55-0.63)$ for patients treated with warfarin; this was lower $(p<0.001)$ than that in the derivation sample. This difference was not unexpected, since as mentioned above, the early kinetics of warfarin anticoagulation differ from those during the initiation of new non-vitamin- $\mathrm{K}$ dependent oral anticoagulants.

TABLE 2 Results of univariate analysis

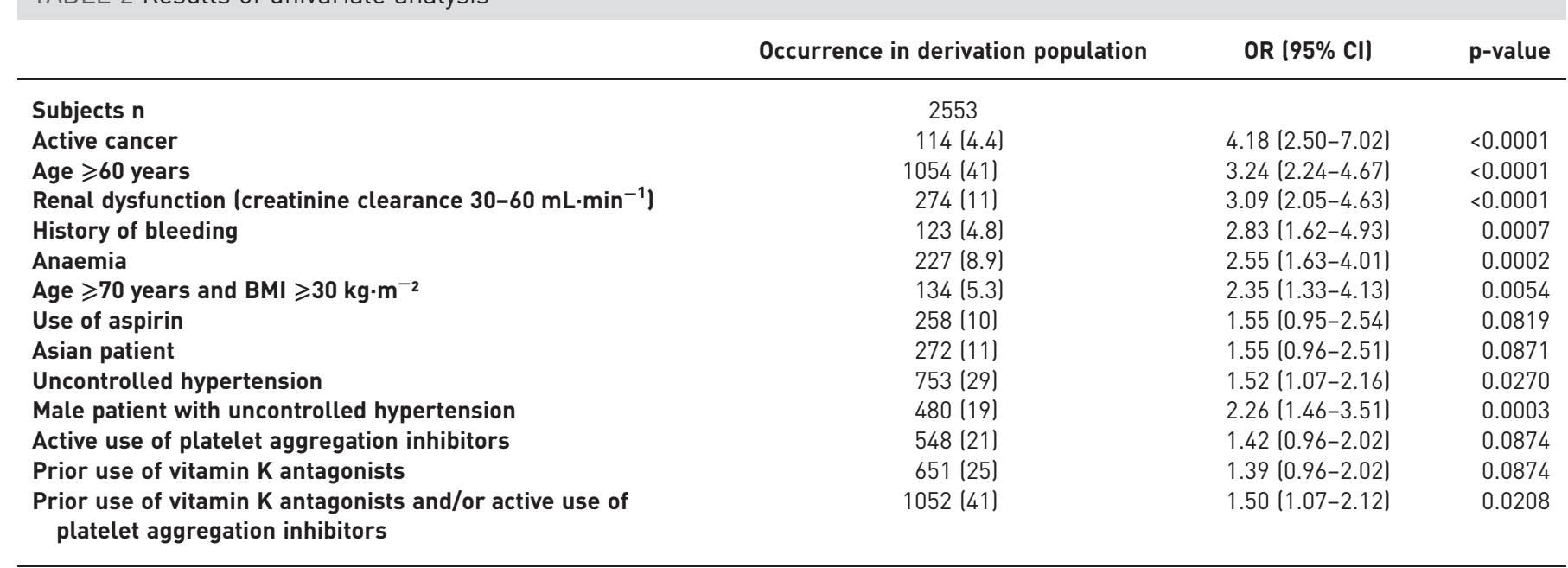

Data are presented as $\mathrm{n}(\%)$, unless otherwise stated. The table shows variables associated with anticoagulation-associated major or clinically relevant non-major bleeding over a 6-month follow-up period in patients who received at least one dose of dabigatran in the RE-COVER studies. Definitions are provided in online supplementary appendix 1 . Only univariate variables with $p<0.1$ are shown. BMI: body mass index. 
TABLE 3 Results of multivariate analysis and score derivation

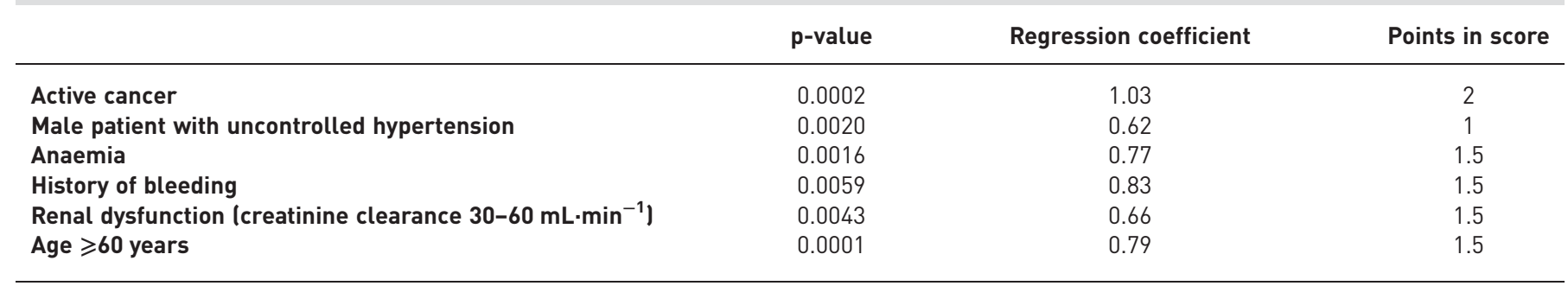

Single individual predictors awarded $<2$ points do not represent a high bleeding risk.

Primary study end-point: performance of the VTE-BLEED score for predicting major bleeding events during stable anticoagulation

During the period between day 30 after randomisation and 6-month follow-up, $13(0.54 \%)$ patients in the dabigatran arm and $25(1.0 \%)$ patients in the warfarin arm had a major bleeding complication (table 1). The c-statistics of our prediction model improved in both treatment arms to 0.75 (95\% CI $0.61-0.89)$ and 0.78 (95\% CI 0.68-0.86) for the dabigatran- and warfarin-treated patients, respectively (fig. 1). For this cohort, a total of $1790(74 \%)$ patients in the dabigatran treatment arm were classified into the low-risk category for major bleeding (incidence $0.22 \%$ ) and $624(26 \%)$ into the elevated-risk category (incidence $1.4 \%$ ). This difference corresponded to an odds ratio of 6.5 (95\% CI 2.0-21) for patients categorised into the elevated bleeding risk category compared to the low-risk category. In the warfarin-allocated cohort, $1800(75 \%)$ patients were classified into the low-risk category (major bleeding risk $0.44 \%)$ and $601(25 \%)$ into the elevated-risk category (major bleeding risk 2.8\%). This also corresponded to an odds ratio of 6.5 (95\% CI 2.8-15).

\section{Performance of the VTE-BLEED score in specific patient subgroups and comparison to existing bleeding scores}

The VTE-BLEED score performed comparably well in the RE-COVER patients randomised to dabigatran who had suffered acute PE and in those with DVT, with c-statistics of 0.74 (95\% CI 0.67-0.80) and 0.71 (95\% CI 0.65-0.76), respectively.

Finally, we determined in the pooled RE-COVER population the point estimates of the c-statistic for existing bleeding scores derived from patients with atrial fibrillation or VTE. All these values were below the lower limit of the $95 \%$ confidence interval of that of the VTE-BLEED score in the derivation cohort (table 4). For the prediction of major bleeding during chronic "stable" anticoagulant treatment, the HAS-BLED, ATRIA and HEMORR ${ }_{2}$ HAGES scores had comparable predictive power.

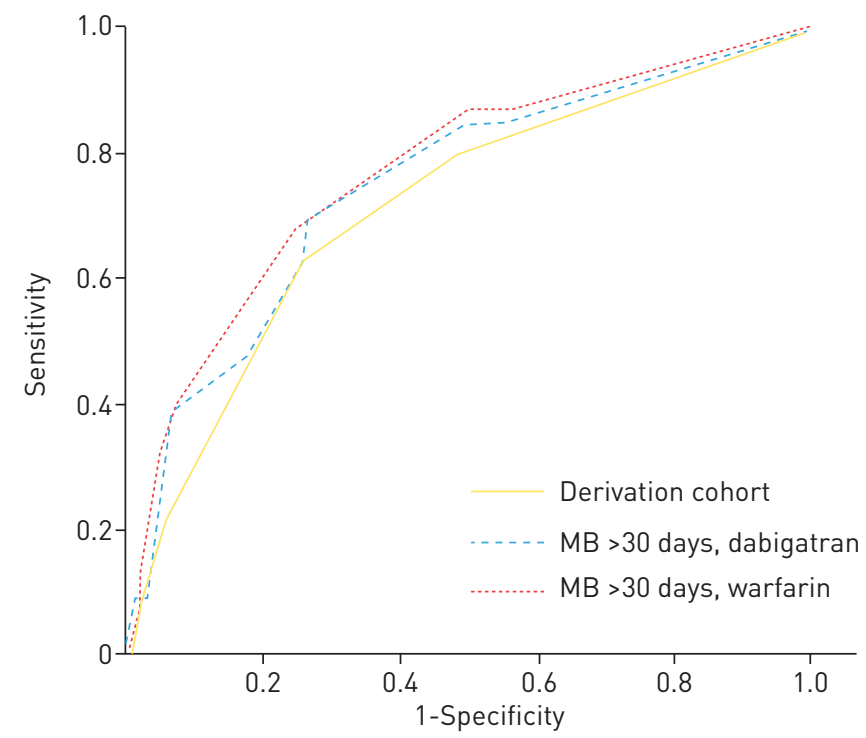

FIGURE 1 Receiver operator characteristic curves for the newly derived VTE-BLEED score for the derivation cohort (dabigatran starting at moment of randomisation) and validation cohorts (warfarin and dabigatran, starting after 30 days from randomisation, focused on major bleeding (MB) only). 
TABLE 4 C-statistics of receiver operator characteristic curves for the new bleeding prediction score derived from the RE-COVER and RE-COVER II trials in comparison to the performance of existing scores for dabigatran-treated patients

\section{C-statistic complete study period ${ }^{\#}$ for dabigatran}

\section{C-statistic "stable" anticoagulation} period" for dabigatran
C-statistic "stable" anticoagulation period" for warfarin

\begin{tabular}{|c|c|c|c|}
\hline VTE-BLEED score & $0.72(0.67-0.76)$ & $0.75(0.61-0.89)$ & $0.78(0.68-0.86)$ \\
\hline Kuijer score [5] & $0.66(0.62-0.71)$ & $0.60(0.4-0.76)$ & $0.68(0.59-0.77)$ \\
\hline OBRI [6] & $0.64(0.59-0.68)$ & $0.69(0.56-0.80)$ & $0.72(0.61-0.81)$ \\
\hline RIETE score [7] & $0.65(0.60-0.70)$ & $0.73(0.59-0.85)$ & $0.65(0.51-0.77)$ \\
\hline ATRIA score [11] & $0.60(0.55-0.64)$ & $0.75(0.63-0.86)$ & $0.73(0.62-0.83)$ \\
\hline HEMORR $_{2}$ HAGES score [12] & $0.63(0.59-0.68)$ & $0.80(0.68-0.90)$ & $0.76(0.68-0.84)$ \\
\hline
\end{tabular}

Data are presented as c-statistic $(95 \% \mathrm{Cl})$. The parameters included in the other scores of bleeding risk, and the definition of risk categories, are shown in online supplementary appendix 2. 95\% confidence intervals were derived from bootstrap analysis. VTE-BLEED: active cancer, male patients with uncontrolled arterial hypertension at baseline, anaemia, history of bleeding, age $\geqslant 60$ years and renal dysfunction; OBRI: outpatient bleeding risk index; RIETE: Registry of Patients with Venous Thromboembolism; HAS-BLED: hypertension, abnormal renal/liver function, stroke, bleeding history or predisposition, labile international normalised ratio, elderly, drugs/alcohol; ATRIA: anticoagulation and risk factors in atrial fibrillation; HEMORR ${ }_{2}$ HAGES: hepatic or renal disease, ethanol abuse, malignancy, older age, reduced platelet count or function, re-bleeding, hypertension, anaemia, genetic factors, excessive fall risk and stroke. "\#: major and non-major clinically relevant bleeding; ${ }^{~}$ : major bleeding only.

\section{Discussion}

The present analysis allowed us to develop a practical and fairly robust clinical score for predicting major bleeding complications during stable oral anticoagulation, either with dabigatran or with warfarin, after an episode of acute VTE. This VTE-BLEED score consists of six simple and objective clinical variables. Importantly, the score appeared to be valid both in patients who presented with DVT and those with PE. Across all patient subgroups and clinical scenarios, the score identified approximately one quarter of the patients who may have an up to 6.5 times higher bleeding risk than the majority of patients who are at low risk. Of note, and according to its label, dabigatran is contraindicated in patients with an eGFR $<30 \mathrm{~mL} \cdot \mathrm{min}^{-1}$; our score is thus not applicable to this specific patient group. Finally, our results suggest the new VTE-BLEED score may outperform bleeding scores previously proposed for VTE. In this regard, it should be noted that bleeding scores derived from and used in patients with atrial fibrillation exhibited comparable performance to VTE-BLEED for predicting major bleeding during the "stable" anticoagulation period in our patient population. This seems consistent with their purpose which is the prediction of bleeding complications during chronic anticoagulant treatment. However, in our opinion, a limitation of those scores as opposed to VTE-BLEED is that they provide more "vague" definitions of at least some of the included variables, and are thus more difficult to reproduce (online supplementary appendix 3).

In contrast to the target-specific mechanism of action of the "new", non-vitamin-K dependent oral anticoagulants, the anticoagulant activity of warfarin depends on the clearance of functional clotting factors from the circulation, with half-lives ranging from $6 \mathrm{~h}$ for factor VII to $72 \mathrm{~h}$ for factor II. Net clearance of these factors starts as early as 1 day after administration of the first warfarin dose, but it may not be fully evident until the fifth day of therapy $[14,19]$. As a result, it may take up to 21 days to achieve stable anticoagulation using warfarin $[14,18]$. Moreover, concomitant initial use of parenteral heparin during titration of vitamin $\mathrm{K}$ antagonists generates an additional bleeding risk during the first days of anticoagulation [4, 14]. Accordingly, the different profile of anticoagulation treatment using warfarin compared to dabigatran may explain, at least in part, the somewhat lower performance of the dabigatran-derived bleeding prediction score in the warfarin arm of the RE-COVER studies when the entire treatment period following randomisation was taken into account. Indeed, the treatment arm related differences in the performance of the score diminished when we focused on the period of stable anticoagulation, during which the mean time in the therapeutic international range of the normalised ratio range was reported to increase from $51 \%$ to $62 \%$ in the RE-COVER studies $[15,16]$.

In accordance with our findings, a recent observational study [9] reported that focusing on the chronic phase of anticoagulation improved the c-statistics of the HAS-BLED score (derived from patients with atrial fibrillation) in a PE cohort. A subanalysis of the EINSTEIN programme confirmed our hypothesis that risk factors for bleeding change over time [20]. In that study, a prediction model for bleeding events was developed and tested for the initial 3 weeks and the period thereafter. Several risk factors overlap between the EINSTEIN score and the VTE-BLEED score, such as age, body weight, sex, cancer and anaemia. Disadvantages of the score proposed by Di Nisio et al. [20] is that it includes the item "rivaroxaban versus enoxaparin/vitamin K antagonist", which is not easily generalisable, and the fact that its performance decreased in the chronic treatment period to a c-statistic of "only" 0.68 (compared to 0.75 and 0.78 for the VTE-BLEED score). 
The VTE-BLEED score was able to identify $25 \%$ of patients with an elevated bleeding risk during chronic anticoagulation, regardless of whether they were treated with dabigatran or warfarin. However, it cannot be ruled out that risk factors for bleeding while on treatment with dabigatran (or another target-specific oral anticoagulant) may slightly differ from those predicting warfarin-associated bleeding. This latter notion appears to be supported by the pooled analysis of phase III trials comparing dabigatran with warfarin for atrial fibrillation or VTE [21]. Similarly, the predictive value of the HAS-BLED and ATRIA scores was reported to deteriorate, compared with the original warfarin-treated derivation cohorts, when they were applied to patients with atrial fibrillation treated with rivaroxaban [22].

We emphasise that the VTE-BLEED score was not designed to contradict or even question the currently established indications for oral anticoagulation, or to interfere with the choice of the oral anticoagulant in patients suffering an episode of acute VTE. Rather than interfering with these issues, this study aimed to address two other unmet medical needs: 1) to identify the minority of patients who may need closer attention while on anticoagulation after VTE; and 2) to provide a tool that can be helpful in the decision-making process regarding the extension of anticoagulant treatment beyond the first 3-6 months. In this sense, our score may be used to complement previously developed clinical decision rules which predict the risk of VTE recurrence after cessation of anticoagulation therapy [23-25]. Optimally, and in addition to further external validation of the VTE-BLEED score, a "net clinical benefit" assessment will be needed to increase the applicability and clinical relevance of our prediction model. This analysis should preferably be in the form of a prospective cohort study focusing on patients with a high VTE-BLEED score and assessing the risks versus benefits of continuing different intensities and drug classes of anticoagulation over longer follow-up periods of $\geqslant 1$ year.

Bleeding complications of oral anticoagulation are more likely to occur in the first days or weeks of treatment. This is particularly true for vitamin $\mathrm{K}$ antagonists, but may also apply to other types of anticoagulant drugs. Accordingly, the decision on whether to extend anticoagulant treatment after the first 3 or 6 months will need to take into consideration the patient's "chronic" bleeding risk, i.e. after the initial phase. This important aspect was the focus of the present paper and will need further validation in external cohorts and with longer follow-up periods.

In our opinion, strong points of the current study are the analysis of high-quality patient-level data from two double-blind randomised clinical trials, which included a large number of patients with very low rates of loss to follow-up and independent adjudication of clinically relevant haemorrhage. In addition, we applied advanced statistical methods, which are in accordance with the recently published TRIPOD (transparent reporting of a multivariable prediction model for individual prognosis or diagnosis) statement [26], and we included only objective variables in the analysis to ensure the applicability of the score in clinical practice. Moreover, we explicitly focused on patients on chronic, stable anticoagulation after VTE. Finally, none of the available bleeding prediction scores have been validated for patients treated with a non-vitamin- $\mathrm{K}$ dependent oral anticoagulant as well as for a vitamin $\mathrm{K}$ antagonist.

The most relevant study limitation is that the new score will also need formal external validation, particularly in a so-called "real-world" cohort of VTE patients, in the period after the initial 6 months of treatment, for other non-vitamin-K dependent oral anticoagulants and for different intensities of long-term anticoagulation. Patients in randomised trials are selected based on strict criteria that, in the case of RE-COVER and the other phase 3 studies on new non-vitamin-K dependent oral anticoagulants, may have excluded patients with the highest risk of bleeding. Furthermore, it is not known whether the risk factors for bleeding in the first 3-6 months can be extrapolated to the extended anticoagulant treatment thereafter, although it is quite likely that these remain very similar. In addition, previously developed bleeding scores used for comparison in the present study were calculated in the RE-COVER population post hoc, which may be a source of bias since not all variables such as "stable INR" were available at baseline (online supplementary appendix 3). Finally, to achieve sufficient statistical power and design a robust multivariate model, we chose to include the complete follow-up period (starting at randomisation) initially as well as both major and clinically relevant non-major bleeding in the derivation analysis, and to subsequently perform a subgroup analysis for major bleeding events only after the first 30 days. The finding that the new score accurately predicted major bleeding during the stable anticoagulation phase in both treatment arms supports the validity of our study design.

In conclusion, we derived a six-variable clinical prediction score that identifies one quarter of patients having an elevated bleeding risk while on chronic anticoagulation treatment after an episode of VTE. The VTE-BLEED score may, if weighed against the individual patient's risk of long-term VTE recurrence, prove helpful in decision making regarding the possible extension of anticoagulation beyond the first 3-6 months. Further research will need to validate this bleeding score outside clinical trial conditions, confirm that it is applicable to oral anticoagulants other than dabigatran or warfarin and ultimately move towards integrating the patient's recurrence and bleeding risk into a single practical decision score. 


\section{Acknowledgements}

All authors have contributed significantly to this manuscript and take responsibility for the analyses. Specifically, F.A. Klok designed the study, interpreted the data and drafted the manuscript; V. Hösel designed the study, performed the analyses, interpreted the data and drafted the manuscript; A. Clemens designed the study, interpreted the data and critically revised the manuscript for important intellectual content; C. Tilke and W.D. Yollo designed and performed the statistical analyses, interpreted the data and critically revised the manuscript for important intellectual content; M. Lankeit designed the study and critically revised the manuscript for important intellectual content; S. Schulman was responsible for data collection and critically revised the manuscript for important intellectual content; S.V. Konstantinides designed the study, interpreted the data and drafted the manuscript.

\section{References}

1 Konstantinides SV, Torbicki A, Agnelli G, et al. 2014 ESC guidelines on the diagnosis and management of acute pulmonary embolism. Eur Heart J 2014; 35: 3033-3069.

2 Kearon C, Akl EA, Comerota AJ, et al. Antithrombotic therapy for VTE disease: antithrombotic therapy and prevention of thrombosis, 9th ed: American College of Chest Physicians evidence-based clinical practice guidelines. Chest 2012; 141: e419S-e494S.

3 Lip GY, Andreotti F, Fauchier L, et al. Bleeding risk assessment and management in atrial fibrillation patients. Thromb Haemost 2011; 106: 997-1011.

4 Klok FA, Kooiman J, Huisman MV, et al. Predicting anticoagulant-related bleeding in patients with venous thromboembolism: a clinically oriented review. Eur Respir J 2015; 45: 201-210.

5 Kuijer PM, Hutten BA, Prins MH, et al. Prediction of the risk of bleeding during anticoagulant treatment for venous thromboembolism. Arch Intern Med 1999; 159: 457-460.

6 Beyth RJ, Quinn LM, Landefeld CS. Prospective evaluation of an index for predicting the risk of major bleeding in outpatients treated with warfarin. Am J Med 1998; 105: 91-99.

7 Ruíz-Giménez N, Suárez C, González R, et al. Predictive variables for major bleeding events in patients presenting with documented acute venous thromboembolism. Findings from the RIETE Registry. Thromb Haemost 2008; 100: $26-31$.

8 Riva N, Bellesini M, Di Minno MN, et al. Poor predictive value of contemporary bleeding risk scores during long-term treatment of venous thromboembolism. A multicentre retrospective cohort study. Thromb Haemost 2014; 112: 511-521.

9 Klok FA, Niemann C, Dellas C, et al. Performance of five different bleeding-prediction scores in patients with acute pulmonary embolism. J Thromb Thrombolysis 2016; 41: 312-320.

10 Pisters R, Lane DA, Nieuwlaat R, et al. A novel user-friendly score (HAS-BLED) to assess 1-year risk of major bleeding in patients with atrial fibrillation: the Euro Heart Survey. Chest 2010; 138: 1093-1100.

11 Fang MC, Go AS, Chang Y, et al. A new risk scheme to predict warfarin-associated hemorrhage: the ATRIA (Anticoagulation and Risk Factors in Atrial Fibrillation) study. J Am Coll Cardiol 2011; 58: 395-401.

12 Gage BF, Yan Y, Milligan PE, et al. Clinical classification schemes for predicting hemorrhage: results from the National Registry of Atrial Fibrillation (NRAF). Am Heart J 2006; 151: 713-719.

13 Kooiman J, van Hagen N, Iglesias Del Sol A, et al. The HAS-BLED score identifies patients with acute venous thromboembolism at high risk of major bleeding complications during the first six months of anticoagulant treatment. PLoS One 2015; 10: e0122520.

14 Ageno W, Gallus AS, Wittkowsky A, et al. Oral anticoagulant therapy: antithrombotic therapy and prevention of thrombosis, 9th ed: American College of Chest Physicians evidence-based clinical practice guidelines. Chest 2012; 141: e44S-e88S.

15 Schulman S, Kakkar AK, Goldhaber SZ, et al. Treatment of acute venous thromboembolism with dabigatran or warfarin and pooled analysis. Circulation 2014; 129: 764-772.

16 Schulman S, Kearon C, Kakkar AK, et al. Dabigatran versus warfarin in the treatment of acute venous thromboembolism. N Engl J Med 2009; 361: 2342-2352.

17 Schulman S, Kearon C. Definition of major bleeding in clinical investigations of antihemostatic medicinal products in non-surgical patients. J Thromb Haemost 2005; 3: 692-694.

18 Kangelaris KN, Bent S, Nussbaum RL, et al. Genetic testing before anticoagulation? A systematic review of pharmacogenetic dosing of warfarin. J Gen Intern Med 2009; 24: 656-664.

19 Hirsh J, Dalen JE, Anderson DR, et al. Oral anticoagulants: mechanism of action, clinical effectiveness, and optimal therapeutic range. Chest 1998; 114: 445S-469S.

20 Di Nisio M, Ageno W, Rutjes AW, et al. Risk of major bleeding in patients with venous thromboembolism treated with rivaroxaban or with heparin and vitamin K antagonists. Thromb Haemost 2016; 115: 424-432.

21 Majeed A, Hwang HG, Connolly SJ, et al. Management and outcomes of major bleeding during treatment with dabigatran or warfarin. Circulation 2013; 128: 2325-2332.

22 Goodman SG, Wojdyla DM, Piccini JP, et al. Factors associated with major bleeding events: insights from the ROCKET AF trial (rivaroxaban once-daily oral direct factor Xa inhibition compared with vitamin K antagonism for prevention of stroke and embolism trial in atrial fibrillation). J Am Coll Cardiol 2014; 63: 891-900.

23 Eichinger S, Heinze G, Jandeck LM, et al. Risk assessment of recurrence in patients with unprovoked deep vein thrombosis or pulmonary embolism: the Vienna prediction model. Circulation 2010; 121: 1630-1636.

24 Marcucci M, Iorio A, Douketis JD, et al. Risk of recurrence after a first unprovoked venous thromboembolism: external validation of the Vienna Prediction Model with pooled individual patient data. J Thromb Haemost 2015; 13: $775-781$.

25 Rodger MA, Kahn SR, Wells PS, et al. Identifying unprovoked thromboembolism patients at low risk for recurrence who can discontinue anticoagulant therapy. CMAJ 2008; 179: 417-426.

26 Collins GS, Reitsma JB, Altman DG, et al. Transparent reporting of a multivariable prediction model for individual prognosis or diagnosis (TRIPOD): the TRIPOD statement. BMJ 2015; 350: g7594. 